\title{
Élaboration de verre plombé radioprotecteur en Colombie : caractérisation des échantillons en fonction de leur concentration d'oxyde de plomb
}

\author{
N. Barbosa ${ }^{1}$, A. Daza ${ }^{1}$, M.C. Plazas ${ }^{1,2 a}$ et D. Paul ${ }^{3}$ \\ 1 Universidad Nacional de Colombia, Groupe de Physique Médicale, Funza, Cundinamarca, Colombia. \\ 2 Instituto de Oncología "Carlos Ardila Lülle", Hospital Universitario Fundación Santa Fe de Bogotá, Funza, Cundinamarca, Colombia. \\ 3 CEA Cadarache, École des Combustibles, 13109 Saint-Paul-lez-Durance, France.
}

Reçu le 16 juillet 2013 - Accepté le 6 janvier 2014

\begin{abstract}
Résumé - La fabrication de verre plombé n'a pas encore été développée en Colombie, ce qui entraîne des lacunes dans la recherche d'améliorations des composants pour la protection radiologique. Le Groupe de Physique Médicale de l'Université Nationale de Colombie a donc initié une étude en vue de développer différents échantillons de verre plombé qui soient adaptés à une utilisation comme blindage contre les rayonnements ionisants. Les caractéristiques optimales ont été trouvées pour une concentration de $50 \%$ d'oxyde de plomb ( $\mathrm{PbO})$ dans un verre de plomb-silicate. Un pourcentage de $\mathrm{PbO}$ supérieur entraîne des difficultés techniques et une diminution de la clarté du verre. Pour cette fraction molaire nous avons trouvé un coefficient d'absorption linéaire de $0,35 \mathrm{~cm}^{-1}$, c'est-à-dire que pour une énergie de $662 \mathrm{keV}$ un centimètre de ce verre correspond à 3,0 $\mathrm{mm}$ de plomb pur; avec cette même énergie la couche de demi-atténuation (CDA) de l'échantillon est égale à celle du ciment ferreux utilisé couramment dans les blindages radiologiques. Enfin il a également été observé que l'application d'une couche d'air de $1 \mathrm{~cm}$ entre les lames de verre diminue la transmission des photons jusqu'à $20 \%$ pour le verre contenant $40 \%$ de plomb.
\end{abstract}

\begin{abstract}
Development of radioprotective lead glass in Colombia: characterization of samples based on their lead oxide concentration. Lead glass production has not yet been developed in Colombia, limiting the improvement of radiological protection elements. In answer to this limitation a number of samples of lead glass with the necessary features for ionizing radiation attenuation have been developed by the Medical Physics Group at the National University of Colombia in Bogota. The optimal characteristics were found in glass with 50\% of lead oxide (PbO). Experiments showed that more than $50 \% \mathrm{PbO}$ results in an opaque glass and is much more difficult to fabricate. For the optimal mole fraction a linear absorption coefficient of $0.35 \mathrm{~cm}^{-1}$ was found. This means, for energy of $662 \mathrm{keV}$, the equivalent thickness of lead to be used would be $3.0 \mathrm{~mm}$. For the same energy, the sample's half-value layer (HVL) is the same as iron concrete, which is commonly used for shielding. Lastly, the use of a 1-cm gap of air between glass layers results in an increase of up to $20 \%$ absorption when $40 \%$ lead-compounded glass is used.
\end{abstract}

Keywords: lead glass / shielding / lead oxide concentration / ionizing radiation / radiological protection

\section{Introduction}

Depuis l'origine, l'être humain a été toujours exposé aux rayonnements, lesquels peuvent être d'origine naturelle : soleil, produits de consommation, entre autres, ou d'origine artificielle : équipements à des fins médicales, industrielles ou autres. Peu de temps après la découverte des rayons X par Roentgen (1895) et la radioactivité par Becquerel (1896), les rayonnements ionisants ont été utilisés pour le diagnostic et le traitement médical. Dans un premier temps, la méconnaissance de l'interaction rayonnement-matière entraîna une pratique irresponsable et rapidement les effets nuisibles apparurent,

\footnotetext{
a mcplazasd@unal.edu.co
}

conséquences d'un haut niveau d'exposition, appelant la mise en place d'une réglementation. En 1934, Mutscheller étudia les effets biologiques de l'exposition aux matières radioactives et établit une dose de tolérance de 3,3 cGy pour des énergies de bas niveau et de 7,2 cGy pour celles de hauts niveaux (Leo, 1994). Du fait qu'il y ait un risque de dégâts biologiques suite à l'exposition aux rayonnements ionisants, il est très important de connaître les moyens les plus efficaces pour se protéger. Nous devons tenir compte des trois grands principes de la radioprotection : la justification qui prévoit qu'une activité ne peut être entreprise que si elle est justifiée pour assurer le fonctionnement normal des installations et de manipulation, l'optimisation qui demande que l'exposition des personnes aux 
rayonnements ionisants soit maintenue au niveau le plus faible possible et la limitation de doses aux travailleurs aux postes de travail exposés, responsabilité des employeurs, qui doivent garantir les niveaux établis par les réglementations nationales à partir des recommandations internationales. L'exposition professionnelle concerne les manipulateurs, les médecins, les radiologues, les physiciens, les infirmières et toute autre personne étant en contact avec des rayonnements ionisants sur son lieu de travail. Les protocoles de protections radiologiques ont évolué tout au long de l'histoire et de nos jours la CIPR publie régulièrement des recommandations.

Parmi les facteurs permettant de limiter la dose nous pouvons faire référence aux temps, distance et blindage. Dans les centres médicaux la distance et le temps de traitement doivent être établis à l'avance par un protocole et le blindage doit permettre une diminution maximale de l'exposition. En Colombie, les normes de protection radiologique du Ministère des Mines et de la Santé permettent de contrôler l'utilisation des blindages dans les centres concernés ${ }^{1}$ (Ministerio de Protección Social de Colombia, 2006).

Dans cet article, nous voulons montrer l'expérience acquise avec la fabrication de verre plombé comme blindage.

Les différents types de réactions entre les rayons $\mathrm{X}$ et $\gamma$ avec la matière : effet photoélectrique, diffusion Compton, production de paires, expliquent les deux principales caractéristiques des photons : 1) les rayons $\mathrm{X}$ et les rayons $\gamma$ sont beaucoup plus pénétrants dans la matière que les particules chargées et 2) un faisceau de photons ne se dégrade pas en énergie au fur et à mesure qu'il traverse une certaine épaisseur de matière, il se dégrade uniquement en intensité en suivant une relation exponentielle comme l'indique la relation de Beer-Lambert (Eq. (1)) :

$$
I=B I_{0} e^{-\mu x}
$$

où $B$ est le facteur d'accumulation en considérant une dispersion pour un faisceau non collimaté et $\mu$ est le coefficient d'atténuation total, tenant compte des 3 types de réactions possibles.

Pour les matériaux composés et les mélanges, le coefficient d'atténuation total peut être calculé par la règle de Bragg (Eq. (2)) :

$$
\frac{\mu}{\rho}=w_{1} \frac{\mu_{1}}{\rho_{1}}+w_{2} \frac{\mu_{2}}{\rho_{2}}+\ldots
$$

où $w_{\mathrm{i}}$ décrit la fraction de chaque élément dans le composant. Le coefficient d'absorption linéaire des composants est déterminé à l'aide du logiciel XCOM créé par NIST ${ }^{2}$. Comme le résultat est donné en $\mathrm{cm}^{2} / \mathrm{g}$ il est important de connaitre la densité de l'échantillon, donnée par la loi d'Archimède référencée à 2 fluides de densités connues (El Batal, 2007).

\footnotetext{
${ }^{1}$ Ministerio de Minas de Colombia : www.minminas.gov.co/ minminas/normatividad.nsf

${ }^{2}$ NIST, http://physics.nist.gov/PhysRefData/Xcom/html/xcom1. html
}

Tableau 1. Epaisseurs des échantillons. Thickness of the samples.

\begin{tabular}{cc} 
& Épaisseur $(\mathbf{c m})$ \\
\hline $40 \%$ & $1,0 \pm 0,1$ \\
$50 \%$ & $1,0 \pm 0,1$ \\
$60 \%$ & $0,9 \pm 0,1$ \\
$70 \%$ & $0,8 \pm 0,1$ \\
\hline
\end{tabular}

\section{Méthodologie de l'élaboration de verre plombé}

Le verre est généralement fabriqué en chauffant un mélange composé de sable siliceux et d'oxydes métalliques secs sous formes pulvérisées ou granulées. Dans le cas du verre plombé, une partie ou la totalité de l'oxyde de calcium est remplacée par du PbO. Lors du processus de fusion, il se crée un liquide visqueux renfermant des bulles et la pâte devient transparente et homogène pour une température proche de 1500 degrés. Une fois sorti du creuset, le verre fondu devient suffisamment rigide pour lui donner la forme souhaitée. Lorsqu'il est refroidi correctement, afin d'éviter la cristallisation, sa viscosité augmente de manière continue. Tout de suite après la mise en forme, il est placé dans un four de recuit continu où il est réchauffé à une température appropriée permettant de supprimer les tensions introduites, avant d'être soumis à un refroidissement lent et contrôlé (Kirk et Othmer, 1962).

Le procédé d'acquisition de nos échantillons a été réalisé de façon rudimentaire, en portant une attention particulière à la durée de chaque étape afin de garantir la qualité du produit final. La procédure suivie est décrite (Fig. 1). On effectue d'abord un mélange à sec (Fig. 1a) : sable siliceux $\left(\mathrm{SiO}_{2}\right)$, carbonate de sodium $\left(\mathrm{Na}_{2} \mathrm{CO}_{3}\right)$, oxyde de calcaire $(\mathrm{CaO})$, feldspath, litharge $(\mathrm{PbO})$. Le mélange est versé dans un creuset réfractaire dans le four de fusion à $1400{ }^{\circ} \mathrm{C}$ (Fig. 1b). Il y reste pendant $2 \mathrm{~h}$. Peu de temps après, les composants acquièrent une texture visqueuse (Fig. 1c). Lorsque le mélange acquiert une texture quasi-liquide, il est versé dans un moule en acier inoxydable (Fig. 1d). Après un court laps de temps, la perte de température entraîne le durcissement du verre et il est alors plus facile de lui donner la forme souhaitée (Fig. 1e). Les verres sont placés immédiatement dans le four de refroidissement pendant 6 heures avec une baisse de température de $1{ }^{\circ} \mathrm{C} / \mathrm{min}$ (Fig. 1f).

\section{Résultats}

\subsection{Les échantillons}

Les échantillons de verre obtenus ont des concentrations de $\mathrm{PbO}$ de $40 \%, 50 \%, 60 \%$ et $70 \%$ (Fig. 2). Les variations d'épaisseurs entre les différents échantillons sont dues à notre processus de fabrication rudimentaire. Les épaisseurs des verres vus sur la figure 1 sont décrites dans le tableau 1 .

Le problème majeur lors de la réalisation du verre est d'éviter les tensions internes qui entraîneraient un verre d'aspect fissuré et peu transparent. Il a été observé que cet effet 


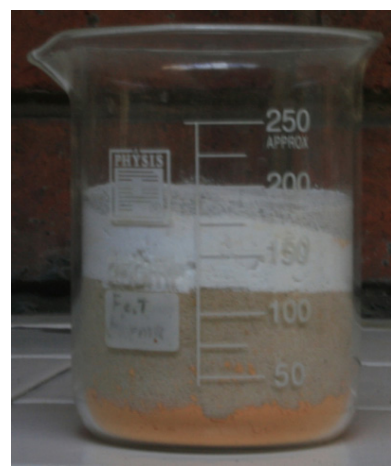

(a)

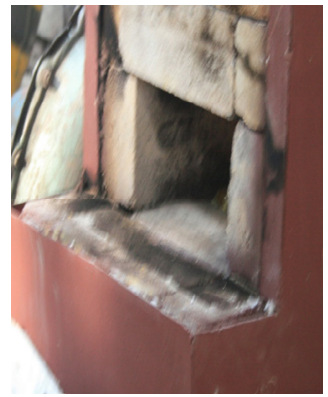

(d)

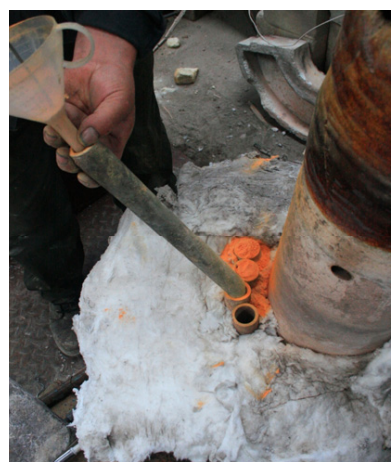

(b)

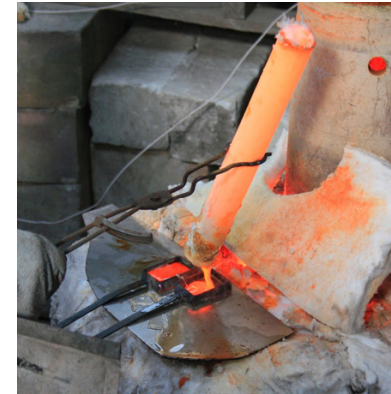

(e)

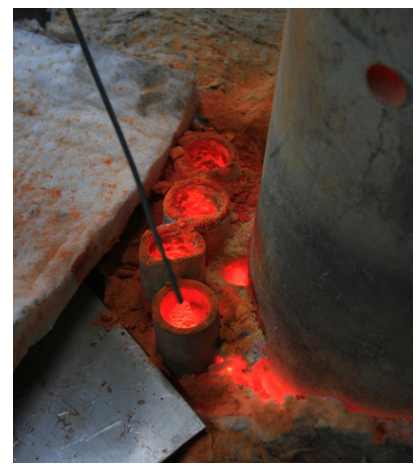

(c)

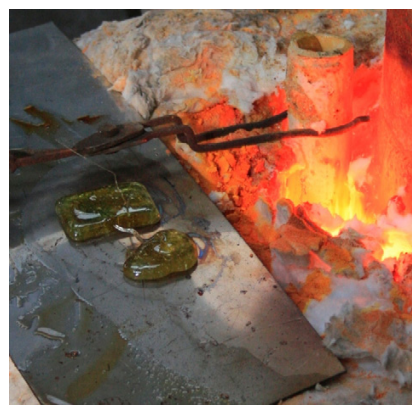

(f)

Fig. 1. Étapes de préparation des échantillons. (a) Mélange, (b,c) four de fusion, (d,e) moulage, (f) four de refroidissement. Samples preparation. (a) Mixture, (b,c) melting furnace, (d,e) molding, (f) cooling furnace.

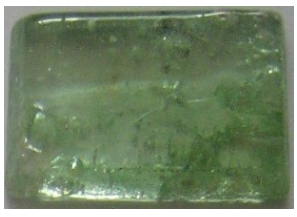

(a)

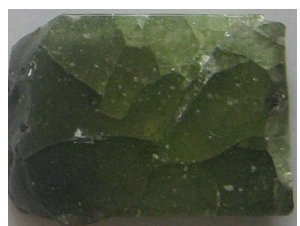

(c)

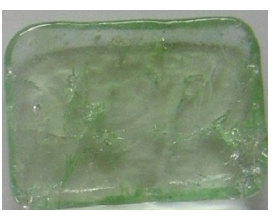

(b)

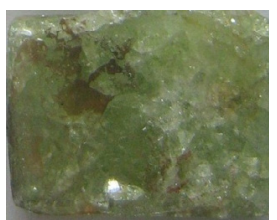

(d)
Fig. 2. Prototype de verres plombés pour différentes concentrations en PbO. (a) $40 \%$, (b) $50 \%$, (c) $60 \%$, (d) $70 \%$.

Lead glass sample for different concentrations of $\mathrm{PbO}$. (a) $40 \%$, (b) $50 \%$, (c) $60 \%$, (d) $70 \%$.

augmentait avec le pourcentage d'oxyde de plomb. Il a également été observé que plus la concentration de $\mathrm{PbO}$ est élevée, plus les échantillons prennent une couleur verte due au fer qui apparaît lors de la fonte et dans le matériau réfractaire (Fig. 2).

Les verres contenant des concentrations de $\mathrm{PbO}$ trop élevées sont inadaptés, car inutilisables du fait de leur opacité. Le verre doit donc contenir un pourcentage élevé de silice et d'autres fondants, et pas uniquement d'oxyde de plomb. Enfin, la rigidité des différents échantillons obtenus a été jugée acceptable pour toutes les concentrations en $\mathrm{PbO}$.

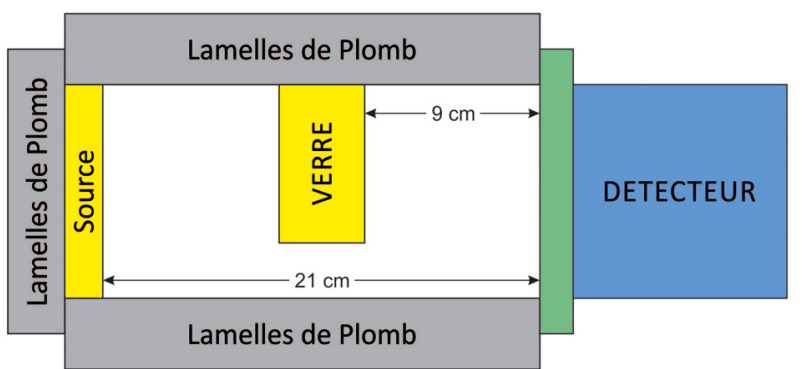

Fig. 3. Schéma du montage expérimental utilisé pour étudier l'atténuation dans les échantillons de verre.

Schema of the setup used to study the glass samples' attenuation.

\subsection{Atténuation au ${ }^{137} \mathrm{Cs}$}

L'atténuation de chaque échantillon a été mesurée à l'aide d'un détecteur à scintillation NaI (Podgorsak, 2005) (résolution de $12,5 \%$ à $662 \mathrm{keV}$ ) et d'une source de ${ }^{137} \mathrm{Cs}$. Ce radio-isotope présente un pic d'énergie unique à $662 \mathrm{keV}$ et une période de 30,23 années. Le montage est détaillé sur la figure 3.

À l'aide de l'équation (1), de l'épaisseur donnée dans le tableau 1 et de la mesure donnée par le compteur $\mathrm{NaI}$ on obtient les données listées dans le tableau 2.

Dans le tableau 2, le facteur d'accumulation $B$ de l'équation (1) n'est pas pris en compte puisque la géométrie du montage ne varie pas. L'atténuation relative des échantillons est donc inchangée. 
Tableau 2. Coefficient d'atténuation linéaire de chaque échantillon. Linear attenuation coefficient for each sample.

\begin{tabular}{ccc}
\hline & $\mathbf{I}_{\mathbf{I}}$ & $\boldsymbol{\mu}(\mathbf{c m})$ \\
\hline Échantillon & 1 & \\
$40 \%$ & 0,744 & $0,29 \pm 0,03$ \\
$50 \%$ & 0,746 & $0,35 \pm 0,04$ \\
$60 \%$ & 0,749 & $0,32 \pm 0,04$ \\
$70 \%$ & 0,758 & $0,34 \pm 0,04$ \\
\hline
\end{tabular}

Tableau 3. Comparaison du coefficient linéaire d'atténuation entre résultats expérimentaux et théoriques ${ }^{1}$.

Comparison of the linear attenuation coefficient between experimental and theoretical results ${ }^{1}$.

\begin{tabular}{cccc} 
Verre & $\begin{array}{c}\text { Résultats } \\
\text { expérimentaux }(\mathbf{c m})\end{array}$ & $\begin{array}{c}\text { Résultats } \\
\text { théoriques }(\mathbf{c m})\end{array}$ & $\begin{array}{c}\text { Différence } \\
(\boldsymbol{\%})\end{array}$ \\
\hline $40 \%$ & $0,29 \pm 0,03$ & $0,34 \pm 0,03$ & 71 \\
$50 \%$ & $0,35 \pm 0,04$ & $0,35 \pm 0,03$ & 0 \\
$60 \%$ & $0,32 \pm 0,04$ & $0,36 \pm 0,03$ & 11 \\
$70 \%$ & $0,34 \pm 0,04$ & $0,37 \pm 0,03$ & 8 \\
\hline
\end{tabular}

Tableau 4. Équivalence en $\mathrm{mm}$ de $\mathrm{Pb}$ pour chaque échantillon. Les équivalences ont été calculées d'après l'équation (1) et à l'aide de mesures avec une sonde NaI.

Equivalence in lead for each sample. The equivalences were calculated from equation (1) and measurements made with a NaI probe.

\begin{tabular}{cc}
\hline Verre & $\begin{array}{c}\text { Equivalent en plomb } \\
(\mathbf{m m})\end{array}$ \\
\hline $40 \%$ & 2,5 \\
$50 \%$ & 3,0 \\
$60 \%$ & 2,5 \\
$70 \%$ & 2,4 \\
\hline
\end{tabular}

Les coefficients d'atténuation linéaires obtenus peuvent être comparés avec les valeurs théoriques données par l'équation (2), en ayant connaissance de la fraction molaire des différents échantillons et à l'aide du programme $\mathrm{XCOM}^{2}$. Le tableau 3 montre les résultats de cette comparaison. Les différences en pourcentages entre résultats théoriques et expérimentaux varient de 0 à $71 \%$, ce qui n'est pas surprenant puisque contrairement à ce qui est supposé dans l'équation (2), les matières premières utilisées n'ont pas un degré de pureté de $100 \%$. Il est cependant important que les valeurs théoriques et expérimentales soient du même ordre de grandeur.

\subsection{Comparaison de l'absorption avec du plomb pur}

Les résultats ont été quantifiés en comparant l'épaisseur de plomb nécessaire pour obtenir la même absorption que celle des échantillons. Pour une énergie de $622 \mathrm{keV}$ le coefficient d'atténuation linéaire pour le plomb est de $\mu=1,16 \mathrm{~cm}^{-12}$. L'équivalence entre les échantillons et une lame de plomb pur est donnée par l'équation (1). Dans le tableau 4, on observe pour un verre d'environ $1 \mathrm{~cm}$ d'épaisseur l'équivalent en plomb pur de chaque échantillon.

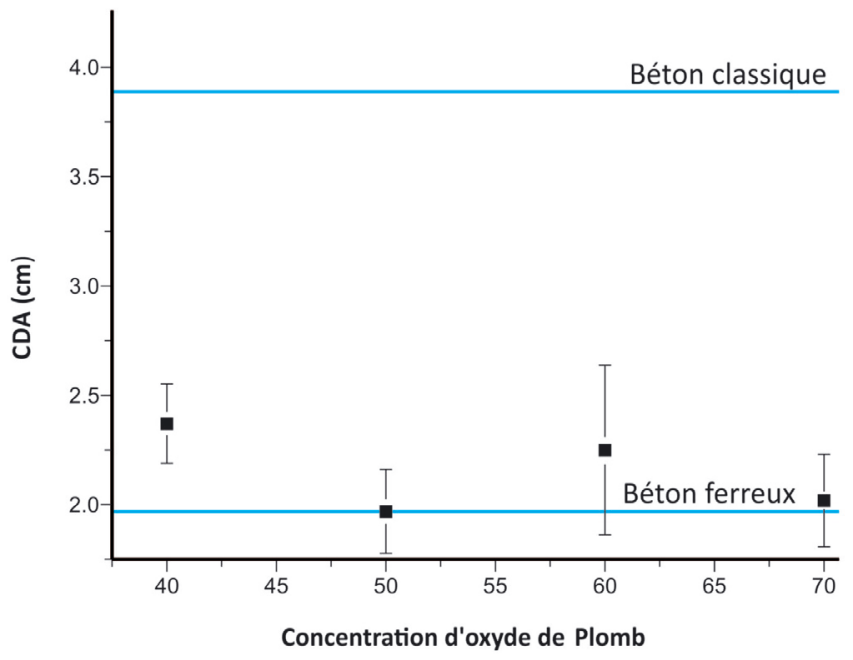

Fig. 4. Comparaison des CDA pour les échantillons de verre, le béton courant et le béton ferreux.

HVL of the glasses compared with common cement and iron.

\subsection{Comparaison de l'absorption avec du béton}

Le béton est couramment utilisé comme matériel pour le blindage radiologique. La CDA (couche de demi-atténuation) de chaque prototype de verre plombé est calculée (Eq. (1)) puis comparé aux CDA du béton commun et du béton ferreux (Fig. 4) (Singh et al., 2008).

Bien que les valeurs de la CDA diminuent lorsque la fraction molaire d'oxyde de plomb augmente, la figure 4 montre une augmentation relative pour le prototype de concentration $60 \%$. Ceci est dû à la diminution du coefficient d'atténuation massique et de la densité de cet échantillon.

Les valeurs de CDA des échantillons de verre sont largement inférieures à celles du béton courant, et semblables à celles du ciment ferreux.

\subsection{Influence de la présence de couches d'air entre les lames de verre}

Sur la figure 5, on observe le pourcentage d'absorption des échantillons pour une fraction molaire de $\mathrm{PbO}$ différente. $\mathrm{La}$ valeur $40 \%-40 \%$ correspond à un blindage de $2 \mathrm{~cm}$ de verre (superposition de 2 lames de verres de $1 \mathrm{~cm}$ d'épaisseur chacune) tandis que $40 \%$-AIR-40\% correspond à deux lames de verres de $1 \mathrm{~cm}$ séparées par une couche d'air de $1 \mathrm{~cm}$ (Fig. 6).

Une augmentation d'environ $20 \%$ est observée lorsque deux lames de verres sont séparées par $1 \mathrm{~cm}$ d'air. La présence d'air permet donc d'augmenter la qualité du blindage avec une perte minimum de transparence et permet de plus d'atténuer les électrons dans le cas de blindage de type double feuillage.

Lorsque la concentration de $\mathrm{PbO}$ augmente, une perte d'atténuation est observée. Cette perte d'atténuation n'est pas due à la réduction de la section efficace mais à la mauvaise qualité de nos échantillons due aux difficultés techniques rencontrées lors du processus de fabrication. 


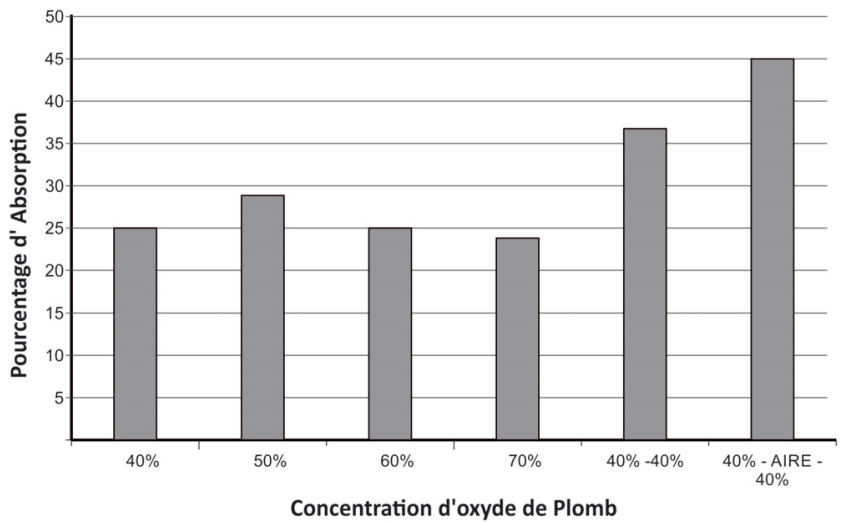

Fig. 5. Absorption en pourcentage dans chacun des échantillons de verre.

Absorption in percent in the glass samples.

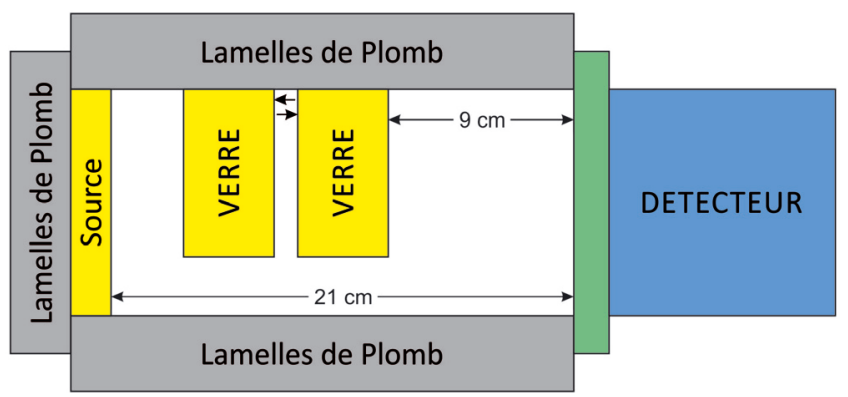

Fig. 6. Schéma du montage expérimental utilisé pour étudier l'influence d'une couche d'air sur les échantillons de verres plombés. Schema of the setup to study the influence of an air gap on the leaded glass samples.

\subsection{Atténuation au ${ }^{99} \mathrm{~m}$ Tc}

En médecine nucléaire, le technétium- $99 \mathrm{~m}\left({ }^{99 \mathrm{~m}} \mathrm{Tc}\right)$ est très utilisé pour l'imagerie diagnostique du fait de sa courte période et de sa faible énergie $(141 \mathrm{keV})$. Le ${ }^{99 \mathrm{~m}} \mathrm{Tc}$ est injecté au patient à l'aide d'une seringue plombée. L'atténuation des échantillons de verre plombé a donc été étudiée pour les photons $\mathrm{du}{ }^{99 \mathrm{~m}} \mathrm{Tc}$.

Le débit d'équivalent de dose a été mesuré avec et sans protection, à l' aide d'un détecteur Geiger-Müller (voir Fig. 3).

Ce débit d'équivalent de dose moyen mesuré sans protection entre la source et le détecteur est de 86,06 $\mu \mathrm{Sv} / \mathrm{h}$. La moyenne relevée pour les différents échantillons de verre radio-protecteur est donnée dans le tableau 5.

Les différents échantillons présentent une bonne absorption, supérieure à $73 \%$, ce qui s'explique par la faible énergie des photons : la section efficace de l'interaction est beaucoup plus élevée que dans le cas précédent $\left({ }^{137} \mathrm{Cs}\right)$ et on observe donc une plus grande atténuation du faisceau par le verre plombé.
Tableau 5. Absorption des photons émis par le ${ }^{99 \mathrm{~m}} \mathrm{Tc}$ pour les différents échantillons de verre.

Absorption of the photons emitted by the ${ }^{99 \mathrm{~m}} \mathrm{Tc}$ for different glass samples.

\begin{tabular}{ccc}
\hline Verre & $\begin{array}{c}\text { Débit d'équivalent de } \\
\text { dose }(\boldsymbol{\mu} \mathbf{S v} / \mathbf{h})\end{array}$ & $\begin{array}{c}\text { Pourcentage } \\
\text { d'absorption }\end{array}$ \\
\hline $40 \%$ & $23,0 \pm 0,1$ & 73,3 \\
$50 \%$ & $18,1 \pm 0,1$ & 78,9 \\
$60 \%$ & $13,8 \pm 0,1$ & 83,9 \\
$70 \%$ & $15,4 \pm 0,1$ & 82,1 \\
\hline
\end{tabular}

Nous remarquons encore que l'absorption varie peu en fonction de la concentration de $\mathrm{PbO}$.

\section{Conclusions}

Les verres avec une concentration élevée d'oxyde de plomb ont l'avantage d'avoir une température de fusion basse mais ils présentent une faible transparence due aux tensions internes et à la présence de fer causant une couleur verdâtre.

Le fait que l'atténuation varie peu selon la concentration de $\mathrm{PbO}$ s'explique par les difficultés techniques rencontrées lors du processus de fabrication, générant une proportion élevée de fondants pour les verres à forte concentration en $\mathrm{PbO}$.

L'échantillon contenant $50 \%$ de $\mathrm{PbO}$ présente les meilleures caractéristiques physiques, la meilleure atténuation et une faible différence avec les résultats théoriques. Son coefficient d'atténuation linéaire est de $0,35 \pm 0,04 \mathrm{~cm}^{-1}: 1 \mathrm{~cm}$ de ce verre correspond à $3,0 \mathrm{~mm}$ de plomb et présente la même CDA que le béton ferreux utilisé couramment dans les blindages.

Une augmentation de $20 \%$ de l'atténuation peut être obtenue pour les verres dont la concentration en oxyde de plomb est égale ou inférieure à $40 \%$ en plaçant une lamelle d'air de $1 \mathrm{~cm}$ entre les deux lamelles de verre plombé.

\section{Références}

El Batal F.H. (2007) Gamma-ray interaction with bismuth silicate glasses, Nucl. Inst. Meth. Phys. Res. B 254, 243-253.

Kirk R., Othmer D. (1962) Enciclopedia de Tecnología Química, Vol. XV.

Leo W.R. (1994) Techniques for Nuclear and Particle Physics Experiments. Springer-Verlag, New York.

Ministerio de Protección Social de Colombia (2006) Manual único de procedimientos de habilitación. Resolución 1043.

Podgorsak E.B. (Ed.) (2005) Radiation Oncology Physics: A Handbook for Teachers and Students. IAEA, Vienna.

Singh K.J. et al. (2008) Gamma-ray shielding and structural properties of $\mathrm{PbO}-\mathrm{SiO}_{2}$ glasses, Nucl. Inst. Meth. Phys. Res. B 266, 944-948. 